\title{
Abstract P5-06-07: PTEN immunohistochemistry is a predictor of mismatch repair status in breast cancer
}

\author{
N Fusco, D Gambini, L Runza, G Lopez, G Ercoli, L Despini and S Bosari
}

DOI: 10.1158/1538-7445.SABCS16-P5-06-07 Published February 2017

\begin{tabular}{ll|} 
- & Article \\
\hline & Info \& Metrics \\
\hline
\end{tabular}

Abstracts: 2016 San Antonio Breast Cancer Symposium; December 6-10, 2016; San Antonio, Texas

\section{Abstract}

Phosphatase and tensin homolog (PTEN), a potent downregulator of the PI3K-Akt pathway, has been shown to mediate the interaction between poly (ADP-ribose) polymerases (PARPs) and the mismatch repair (MMR) complex in endometrial and ovarian cancer. Drugs inhibiting PARPs (iPARPs) are currently considered promising therapeutic tools in a subset of PTEN-defective tumors. Regrettably, the frequency and significance of MMR alterations in breast cancer is debated, and their relationship with PTEN status has not been investigated in the breast. Furthermore, many of the studies on the DNA damage response and its therapeutic implications in breast cancer focus on inherited syndromes (e.g. Lynch syndrome and hereditary breast-ovarian cancer syndrome).

Aims: We sought to explore the interplay between PTEN and the MMR system and to define whether PTEN immunohistochemistry (IHC) is a predictor of MMR proficiency in non-familial breast cancers. Methods: 373 cases of non-familial breast cancers, including a representative number of no special $(n=295)$ and special types $(n=78)$, carefully characterized from clinical and pathological standpoints, were reviewed and used to construct 14 tissue microarrays (TMAs). For each case, a mean of 4.5 tumor tissue cores (range 3 to 6 cores) was sampled, incorporating distinct topographic areas of the tumor, as well as matched non-neoplastic breast tissue. Taken together, 1876 spots were generated. Each TMA was subjected to IHC for PTEN and the DNA MMR proteins MLH1, MSH2, MSH6 and PMS2. In order to minimize human-related biases, each stained slide was digitalized and two pathologists blindly analyzed each tumor spot using a dedicated software able to segment and randomize TMA cores. The pattern of expression was therefore annotated manually on a digital database using a specific add-on module to reconstruct the original topography.

Results: According to clinicopathologic surrogate definition of intrinsic subtypes, PTEN protein loss or heterogeneous expression was more frequent in estrogen receptor negative cancers. Furthermore, $100 \%$ of the MMR-proficient luminal B-like (HER2+) and triple-negative breast cancers displayed strong and diffuse homogeneous PTEN expression, while PTEN-positive status identified MMR-proficient luminal A-like and luminal B (HER2-) like tumors with accuracy rates of $89.3 \%$ and $92.7 \%$, respectively $(p=0.001$, Fisher's exact test).

Conclusions: The present study is the first to investigate PTEN protein loss in a large set of non-familial breast carcinomas based on their DNA MMR status by IHC. Here, we demonstrated that PTEN strong and homogeneous expression by $\mathrm{IHC}$ is able to capture the vast majority of MMR-proficient non-familial breast cancers. Our findings broaden the understanding of the biology underpinning these tumors, suggesting that PTEN is likely play a role in the development of MMR alterations. Given that PTENdefective breast cancers have the propensity to develop additional somatic alterations in the MMR system, our results suggest that IHC for PTEN and MMR proteins may be emplyed as an ancillary study to define new subclasses of sporadic breast cancers potentially eligible for iPARPs therapies. Citation Format: Fusco N, Gambini D, Runza L, Lopez G, Ercoli G, Despini L, Bosari S. PTEN immunohistochemistry is a predictor of mismatch repair status in breast cancer [abstract]. In: Proceedings of the 2016 San Antonio Breast Cancer Symposium; 2016 Dec 6-10; San Antonio, TX. Philadelphia (PA): AACR; Cancer Res 2017;77(4 Suppl):Abstract nr P5-06-07. 


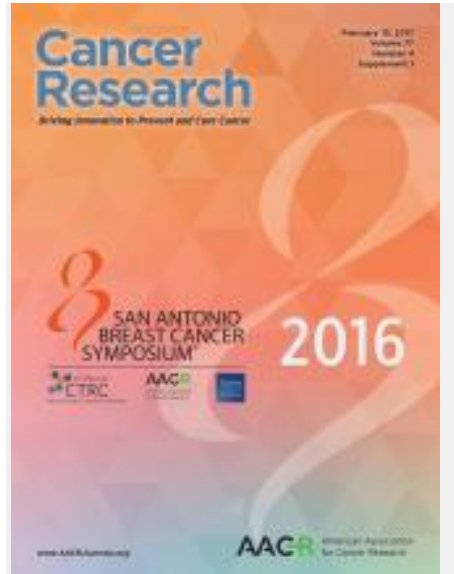

February 2017

Volume 77, Issue 4 Supplement

- Table of Contents

Search for this keyword 